\title{
The momentum distribution of two bosons in one dimension with infinite contact repulsion in harmonic trap gets analytical
}

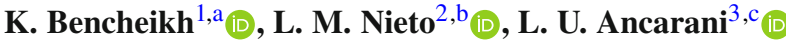 \\ ${ }^{1}$ Département de Physique. Laboratoire de physique quantique et systèmes dynamiques, Université Ferhat \\ Abbas Sétif-1, 19000 Setif, Algeria \\ 2 Departamento de Física Teórica, Atómica y Óptica and IMUVA, Universidad de Valladolid, 47011 Valladolid, \\ Spain \\ 3 UMR 7019, LPCT, Université de Lorraine-CNRS, 57000 Metz, France
}

Received: 6 March 2021 / Accepted: 13 June 2021

(C) The Author(s) 2021

\begin{abstract}
For a harmonically trapped system consisting of two bosons in one spatial dimension with infinite contact repulsion (hard core bosons), we derive an expression for the onebody density matrix $\rho_{\mathrm{B}}$ in terms of center of mass and relative coordinates of the particles. The deviation from $\rho_{\mathrm{F}}$, the density matrix for the two fermions case, can be clearly identified. Moreover, the obtained $\rho_{\mathrm{B}}$ allows us to derive a closed form expression of the corresponding momentum distribution $n_{\mathrm{B}}(p)$. We show how the result deviates from the noninteracting fermionic case, the deviation being associated with the short-range character of the interaction. Mathematically, our analytical momentum distribution is expressed in terms of one and two variables confluent hypergeometric functions. Our formula satisfies the correct normalization and possesses the expected behavior at zero momentum. It also exhibits the high momentum $1 / p^{4}$ tail with the appropriate Tan's coefficient. Numerical results support our findings.
\end{abstract}

\section{Introduction}

Two-body models have the merit that they can be solved exactly and give direct access to the wave function and to the one-body density matrix. We consider here two bosonic atoms with mass $m$ in one dimension with infinite repulsive contact interaction (two hard core bosons or the so-called Tonks-Girardeau regime). The system is also subjected to an external harmonic potential trap with frequency $\omega$. A system of Tonks-Girardeau (TG) gas has been already realized in ultra-cold experiments [1]. On the theoretical side, the TG model is exactly solvable through the Bose-Fermi mapping theorem, which relates this gas to a system of noninteracting spin polarized fermions [2,3]. The two systems-bosonic and fermionicexhibit some identical properties such as the particle density. However, their corresponding one-body density matrices are not the same resulting into a considerable difference between

\footnotetext{
a e-mail: bencheikhkml@univ-setif.dz

b e-mail: luismiguel.nieto.calzada@uva.es (corresponding author)

c e-mail: ugo.ancarani@univ-lorraine.fr
} 
the momentum density of TG gas from that of an ideal Fermi gas [4,5]. In an exhaustive study, Forrester et al [6] have examined the properties of untrapped and also of harmonically trapped arbitrary $N$ TG bosons, and in particular they managed to give closed expression of one-body density matrix in terms of Hankel type determinants. Very recently, in an interesting study carried out on a finite temperature harmonically trapped one-dimensional gas, the case of two identical bosons was examined and the way to calculate the canonical density matrix of the last system was clearly shown [7]. While it is rather easy to calculate the momentum density of an ideal system of fermions, the task is difficult for a TG gas [4]. The main purpose of the present work is to provide a definitive solution for the case of two particles, deriving a closed form expression of the momentum distribution at zero temperature for two harmonically trapped interacting bosons in the TG regime. The analytical approach allows us to clearly identify the deviation from the fermionic counterpart.

Let $\psi_{\mathrm{B}}\left(x_{1}, x_{2}\right)$ denote the ground state wave function of the system of two harmonically trapped TG atoms, corresponding to the Hamiltonian

$$
H=-\frac{\hbar^{2}}{2 m}\left(\frac{\partial^{2}}{\partial x_{1}^{2}}+\frac{\partial^{2}}{\partial x_{2}^{2}}\right)+\frac{m \omega^{2}}{2}\left(x_{1}^{2}+x_{2}^{2}\right),
$$

with impenetrability condition $\psi_{\mathrm{B}}\left(x_{1}, x_{2}\right)=0$ when $x_{2}=x_{1}$. According to the Bose-Fermi mapping theorem [3], we can write

$$
\psi_{\mathrm{B}}\left(x_{1}, x_{2}\right)=\left|\psi_{F}\left(x_{1}, x_{2}\right)\right|
$$

where $\psi_{F}\left(x_{1}, x_{2}\right)$ is the fermionic ground state, that is, a Slater determinant

$$
\psi_{F}\left(x_{1}, x_{2}\right)=\frac{1}{\sqrt{2}}\left(\varphi_{0}\left(x_{1}\right) \varphi_{1}\left(x_{2}\right)-\varphi_{0}\left(x_{2}\right) \varphi_{1}\left(x_{1}\right)\right) .
$$

built on the two lowest single particle normalized eigenfunctions $\varphi_{0}(x)$ and $\varphi_{1}(x)$ of the harmonic oscillator potential trap. Explicitly we have

$$
\varphi_{0}(x)=\sqrt{\frac{1}{\ell \sqrt{\pi}}} e^{-\frac{1}{2}(x / \ell)^{2}}, \quad \varphi_{1}(x)=\sqrt{\frac{2}{\ell \sqrt{\pi}}} \frac{x}{\ell} e^{-\frac{1}{2}(x / \ell)^{2}},
$$

where $\ell=\sqrt{\hbar / m \omega}$ denotes the harmonic oscillator characteristic length. After insertion in (2), we get

$$
\psi_{\mathrm{B}}\left(x_{1}, x_{2}\right)=\frac{1}{\ell \sqrt{\pi}} e^{-\frac{1}{2}\left(\left(x_{1} / \ell\right)^{2}+\left(x_{2} / \ell\right)^{2}\right)}\left|\frac{x_{1}}{\ell}-\frac{x_{2}}{\ell}\right|=\psi_{\mathrm{B}}\left(x_{2}, x_{1}\right) .
$$

A few words about this wave function are in order. It may also be considered in the context of one-dimensional spinor quantum gases studies, a topic which has recently received a lot of attention [8-10]. Let us consider the particular case of two ultra-cold bosons in the Tonks-Girardeau regime occupying or prepared in two hyperfine states; this constitutes a pseudo-spin-1/2 system of different spins (which we denote by $\uparrow$ and $\downarrow)$, the wave function $\psi_{\mathrm{B}}\left(x_{1}, x_{2}\right)=\left|\psi_{F}\left(x_{1}, x_{2}\right)\right|$ in (5) being the spatial wave function of a spin-1/2 hardcore bosons corresponding to the full wave function of the system, $\Phi=\left|\psi_{F}\left(x_{1}, x_{2}\right)\right| \otimes \chi^{S=1}$, where $\chi^{S=1}=(|\uparrow \downarrow\rangle+|\downarrow \uparrow\rangle) / \sqrt{2}$ is the triplet (symmetric) spin state [11]. Another possible configuration [11] would be to use a singlet (antisymmetric) spin state $\chi^{S=0}=(|\uparrow \downarrow\rangle-|\downarrow \uparrow\rangle) / \sqrt{2}$. In this case, the full wave function would simply read $\Psi=\psi_{F}\left(x_{1}, x_{2}\right) \otimes \chi^{S=0}$, and calculations are very easy. In the present investigation, we consider the much more difficult triplet spin state case for which the calculations are by far not straightforward because of the presence of the absolute value in the wave function. 
The plan of the work is the following. In Sect. 2, we look at the zero temperature one-body density matrix, denoted here $\rho_{\mathrm{B}}\left(x_{1}, x_{2}\right)$, that corresponds to the two-particle wave function (5). An explicit expression, given in terms of the physical positions, has been reported long ago in [5,6] and also more recently in [12]; it involves the function $\operatorname{sign}\left(x_{1}-x_{2}\right)$ which, in this context, can be conveniently substituted by the absolute value making mathematical expressions more tractable. We propose here an alternative and novel expression, given in terms of the coordinate of the center of mass and the relative coordinate (more precisely the relative distance $\left.\left|x_{1}-x_{2}\right|\right)$. This expression, which represents the first finding of our investigation, results to be more suitable for practical use for two reasons:

(i) At this level, if one is interested in the short-range behavior of the density matrix, it is obtained directly through a Taylor expansion of our density matrix in terms of powers of the distance $\left|x_{1}-x_{2}\right|$. Let us mention that this short distance expansion leads to direct access to the asymptotic behavior of the momentum distribution at high values of the momentum [see for instance [13]].

(ii) As we will see later, the momentum distribution is a physical quantity that incorporates the one-body properties of the gas; it can be shown to be given as the Fourier transform, with respect to the relative coordinate, of a function proportional to the density matrix. Our alternative expression of the density matrix leads to an easier evaluation of such momentum distribution. Indeed, in Sect. 3 we are able to derive a closed form for the momentum density which holds for arbitrary values of the momentum $p$.

This second analytical result is analyzed and tested in Sect. 4 along three lines: (i) we check that one recovers the correct value of the momentum density at $p=0$; (ii) we check that our density satisfies the required normalization condition $\int n_{\mathrm{B}}(p) \mathrm{d} p=2$; (iii) we prove analytically, and we verify numerically, that our momentum distribution exhibits a well-known result in quantum gases with contact repulsive interactions [13], namely that the momentum distribution of the atoms decays asymptotically as $1 / p^{4}$ for large momentum $p$, and as a consequence we recover the exact expression of the so-called Tan's contact coefficient for the case of two interacting particles [14]. In the last section, the results of the paper are summarized.

\section{One-body density matrix of a one-dimensional system of two bosons with infinite contact repulsion in harmonic trap}

The one-body density matrix for the system under study is given by [15]

$$
\rho_{\mathrm{B}}\left(x_{1}, x_{2}\right)=2 \int_{-\infty}^{+\infty} \psi_{\mathrm{B}}\left(x_{1}, x^{\prime}\right) \psi_{\mathrm{B}}^{*}\left(x_{2}, x^{\prime}\right) \mathrm{d} x^{\prime} .
$$

Using the explicit expression (5) and denoting $z_{1}=x_{1} / \ell, z_{2}=x_{2} / \ell, \xi=x^{\prime} / \ell$, the density can be written as

$$
\begin{aligned}
\rho_{\mathrm{B}}\left(x_{1}, x_{2}\right) & =\frac{2}{\ell \pi} e^{-\frac{1}{2}\left(z_{1}^{2}+z_{2}^{2}\right)} \int_{-\infty}^{\infty} e^{-\xi^{2}}\left|z_{1}-\xi\right|\left|z_{2}-\xi\right| \mathrm{d} \xi \\
& =\frac{2}{\ell \pi} e^{-\frac{1}{2}\left(z_{1}^{2}+z_{2}^{2}\right)} \int_{-\infty}^{\infty} e^{-\xi^{2}}\left|(\xi-u)^{2}-v^{2}\right| \mathrm{d} \xi,
\end{aligned}
$$

where $u$ is the dimensionless coordinate of the center of mass of the two particles and $v$ is half the dimensionless relative coordinate of the two particles:

$$
u=\frac{z_{1}+z_{2}}{2}=\frac{x_{1}+x_{2}}{2 \ell}, \quad v=\frac{z_{1}-z_{2}}{2}=\frac{x_{1}-x_{2}}{2 \ell} .
$$


Performing now the change of variable $w=(\xi-u)$, Eq. (7) takes the form

$$
\rho_{\mathrm{B}}\left(x_{1}, x_{2}\right)=\frac{2}{\ell \pi} e^{-\left(u^{2}+v^{2}\right)} \int_{-\infty}^{\infty} e^{-(w+u)^{2}}\left|w^{2}-v^{2}\right| \mathrm{d} w .
$$

To carry out the above integration, we remove the absolute value symbol and write the density matrix as

$$
\rho_{\mathrm{B}}\left(x_{1}, x_{2}\right)=\frac{2}{\ell \pi} e^{-\left(u^{2}+v^{2}\right)} \mathcal{A}(u, v), \quad \mathcal{A}(u, v)=\mathcal{B}(u, v)-\mathcal{C}(u, v)+\mathcal{B}(-u, v),
$$

where

$$
\mathcal{B}(u, v)=\int_{-\infty}^{-|v|} e^{-(w+u)^{2}}\left(w^{2}-v^{2}\right) \mathrm{d} w, \quad \mathcal{C}(u, v)=\int_{-|v|}^{|v|} e^{-(w+u)^{2}}\left(w^{2}-v^{2}\right) \mathrm{d} w .
$$

The three functions $\mathcal{A}(u, v), \mathcal{B}(u, v)$ and $\mathcal{C}(u, v)$ are calculated in Appendix $\mathrm{A}$ in terms of the error function $\operatorname{erf}(x)=2 / \sqrt{\pi} \int_{0}^{x} e^{-t^{2}} \mathrm{~d} t$. Substituting the result (A7) into (10), we obtain the one-body density matrix $\rho_{\mathrm{B}}\left(x_{1}, x_{2}\right)$ in the form

$$
\begin{aligned}
\rho_{\mathrm{B}}\left(x_{1}, x_{2}\right)= & \frac{2}{\ell \pi} e^{-\left(u^{2}+v^{2}\right)}\left\{(u+|v|) e^{-(u-|v|)^{2}}-(u-|v|) e^{-(u+|v|)^{2}}\right. \\
& \left.+\sqrt{\pi}\left(\frac{1}{2}-v^{2}+u^{2}\right)[1+\operatorname{erf}(u-|v|)-\operatorname{erf}(u+|v|)]\right\} .
\end{aligned}
$$

For two noninteracting fermions, the one-body density matrix is given by

$$
\rho_{F}\left(x_{1}, x_{2}\right)=\varphi_{0}\left(x_{1}\right) \varphi_{0}^{*}\left(x_{2}\right)+\varphi_{1}\left(x_{1}\right) \varphi_{1}^{*}\left(x_{2}\right)=\frac{2}{\ell \sqrt{\pi}} e^{-\left(u^{2}+v^{2}\right)}\left(\frac{1}{2}-v^{2}+u^{2}\right),
$$

where we have used the explicit expressions (4) of $\varphi_{0}(x), \varphi_{1}(x)$. Remark that these densities are correctly normalized to the particle number, so that $\int \rho_{\mathrm{B}}(x, x) \mathrm{d} x=\int \rho_{F}(x, x) \mathrm{d} x=2$. By comparing the bosonic to the fermionic density, we may write

$$
\rho_{\mathrm{B}}\left(x_{1}, x_{2}\right)=\rho_{F}\left(x_{1}, x_{2}\right)+\rho_{D}\left(x_{1}, x_{2}\right)
$$

and thus clearly identify the deviation

$$
\begin{aligned}
\rho_{D}\left(x_{1}, x_{2}\right)= & \frac{2}{\ell \pi}[2 u \sinh (2 u|v|)+2|v| \cosh (2 u|v|)] e^{-2 u^{2}} e^{-2 v^{2}} \\
& +\frac{2}{\ell \sqrt{\pi}}\left(\frac{1}{2}-v^{2}+u^{2}\right)[\operatorname{erf}(u-|v|)-\operatorname{erf}(u+|v|)] e^{-u^{2}} e^{-v^{2}} .
\end{aligned}
$$

Note that if $x_{1}=x_{2}=x$ then $u=x, v=0$, and we obtain $\rho_{D}(x, x)=0$ and therefore $\rho_{\mathrm{B}}(x, x)=\rho_{F}(x, x)$, as required by the Bose-Fermi mapping theorem [3].

Expression (14), together with the explicit deviation (15), constitutes the first outstanding result of the present study. It should be pointed out that, although the Tonks-Girardeau gas and the ideal Fermi gas have identical local spatial density $\rho_{\mathrm{B}}(x)=\rho_{F}(x)$, they display different one-body density matrices yielding to very different momentum density profiles [16]. In the sequel, this property will be explicitly shown and analytically verified in the case of two particles.

Before proceeding further, it is interesting to note that, at this level, one can perform an expansion of the density matrix in (14) with (15) in terms of $|v|=\left|x_{1}-x_{2}\right| /(2 \ell)$. This corresponds to a short-distance expansion and allows one to directly access to momentum density profile at momentum high-values [17]. This asymptotic behavior is of particular interest in the study of short-range interacting systems [18]. 


\section{Closed-form expression of the momentum distribution}

In what follows, we will derive an analytical expression of the momentum distribution for the system of two bosons in the Tonks-Girardeau regime. In terms of the one-body density matrix, the momentum density denoted by $n_{\mathrm{B}}(p)$ is given by [15]

$$
n_{\mathrm{B}}(p)=\frac{1}{2 \pi \hbar} \int_{-\infty}^{\infty} \int_{-\infty}^{\infty} \rho_{\mathrm{B}}\left(x_{1}, x_{2}\right) e^{-i p\left(x_{1}-x_{2}\right) / \hbar} \mathrm{d} x_{1} \mathrm{~d} x_{2} .
$$

and is normalized to the total particle number, i.e., $\int n_{\mathrm{B}}(p) \mathrm{d} p=2$. In terms of center of mass and relative dimensionless coordinates $(u, v)$, we have

$$
n_{\mathrm{B}}(p)=\frac{\ell^{2}}{\pi \hbar} \int_{-\infty}^{\infty} \int_{-\infty}^{\infty} \rho_{\mathrm{B}}(\ell(u+v), \ell(u-v)) e^{-2 i \ell p v / \hbar} \mathrm{d} u \mathrm{~d} v .
$$

As indicated in the Introduction, this expression can be seen as the Fourier transform with respect to the relative coordinate. Now, substituting (14) into (17), we can write

$$
n_{\mathrm{B}}(p)=n_{F}(p)+n_{D}(p)
$$

with

$$
n_{F}(p)=\frac{2 \ell}{\sqrt{\pi} \pi \hbar} \int_{-\infty}^{\infty} \int_{-\infty}^{\infty}\left(\frac{1}{2}-v^{2}+u^{2}\right) e^{-u^{2}} e^{-v^{2}} e^{-2 i \ell p v / \hbar} \mathrm{d} u \mathrm{~d} v,
$$

which is nothing but the momentum density of two noninteracting fermions in harmonic trap, and

$$
\begin{aligned}
n_{D}(p)= & I_{1}+I_{2}=\frac{4 \ell}{\pi^{2} \hbar} \int_{-\infty}^{\infty} \int_{-\infty}^{\infty}(|v| \cosh (2 u|v|)+u \sinh (2 u|v|)) e^{-2 u^{2}} e^{-2 v^{2}} e^{-2 i \ell p v / \hbar} \mathrm{d} u \mathrm{~d} v \\
& +\frac{2 \ell}{\pi^{3 / 2} \hbar} \int_{-\infty}^{\infty} \int_{-\infty}^{\infty}\left(\frac{1}{2}-v^{2}+u^{2}\right)(\operatorname{erf}(u-|v|)-\operatorname{erf}(u+|v|)) e^{-u^{2}} e^{-v^{2}} e^{-2 i \ell p v / \hbar} \mathrm{d} u \mathrm{~d} v
\end{aligned}
$$

which is the momentum density corresponding to the deviation $\rho_{D}$. Next we are going to evaluate the integrals in (19)-(20). It will be convenient to use the momentum-dependent dimensionless variable $q=\ell p / \hbar$.

\subsection{Evaluation of $n_{F}(p)$}

The integration with respect to $u$ in (19) yields

$$
n_{F}(p)=\frac{4 \ell}{\hbar \pi}\left[\int_{0}^{\infty} e^{-v^{2}} \cos (2 q v) \mathrm{d} v-\int_{0}^{\infty} v^{2} e^{-v^{2}} \cos (2 q v) \mathrm{d} v\right]
$$

By using eq. (3.952), number (9) of ref. [19]

$$
\int_{0}^{\infty} x^{2 n} e^{-\beta^{2} x^{2}} \cos (\alpha x) \mathrm{d} x=\frac{(-1)^{n} \sqrt{\pi}}{(2 \beta)^{2 n+1}} e^{-\frac{\alpha^{2}}{4 \beta^{2}}} H_{2 n}\left(\frac{\alpha}{2 \beta}\right),
$$

the integrals in (21) are easily computed, giving the final expression of the momentum density $n_{F}(p)$ :

$$
n_{F}(p)=\frac{\ell}{\sqrt{\pi} \hbar}\left(1+2 q^{2}\right) e^{-q^{2}} .
$$


It is easy to check that it is normalized as $\int n_{F}(p) \mathrm{d} p=2$. The same $n_{F}(p)$ result can be derived also by using the expression, $n_{F}(p)=\left|\widetilde{\varphi}_{0}(p)\right|^{2}+\left|\widetilde{\varphi}_{1}(p)\right|^{2}$, where $\widetilde{\varphi}_{0,1}(p)$ denote the single particle wave functions in momentum representation, i.e., the Fourier transforms

$$
\widetilde{\varphi}_{0,1}(p)=\frac{1}{\sqrt{2 \pi \hbar}} \int \varphi_{0,1}(x) e^{-i \frac{p}{\hbar} x} \mathrm{~d} x
$$

of the single particle wave functions $\varphi_{0,1}(x)$ [20].

\subsection{Evaluation of $n_{D}(p)$}

Let us start with the second term of the first double integral $I_{1}$ in (20), and perform an integration by parts with respect to $u$, to get

$$
\begin{aligned}
& \int_{-\infty}^{\infty} \int_{-\infty}^{\infty} u \sinh (2 u|v|) e^{-2 u^{2}} e^{-2 v^{2}} e^{-2 i q v} \mathrm{~d} u \mathrm{~d} v \\
& =\frac{1}{2} \int_{-\infty}^{\infty} \int_{-\infty}^{\infty}|v| \cosh (2 u|v|) e^{-2 u^{2}} e^{-2 v^{2}} e^{-2 i q v} \mathrm{~d} u \mathrm{~d} v
\end{aligned}
$$

Combining now the two terms, and integrating over $u$ (simple Gaussian-type integral [19]), the first double integral in (20) simplifies into

$$
\begin{aligned}
I_{1} & =\frac{6 \ell}{\pi^{2} \hbar} \int_{-\infty}^{\infty} \int_{-\infty}^{\infty}|v| \cosh (2 u|v|) e^{-2 u^{2}} e^{-2 v^{2}} e^{-2 i q v} \mathrm{~d} u \mathrm{~d} v \\
& =\frac{6 \ell \sqrt{2}}{\pi^{3 / 2} \hbar} \int_{0}^{\infty} v e^{-\frac{3}{2} v^{2}} \cos (2 q v) \mathrm{d} v .
\end{aligned}
$$

For the second double integral $I_{2}$ in (20), we use the property $\operatorname{erf}(-x)=-\operatorname{erf}(x)$, to write

$$
I_{2}=\frac{4 \ell}{\pi^{3 / 2} \hbar} \int_{-\infty}^{\infty} e^{-v^{2}} \cos (2 q v) \mathrm{d} v \int_{-\infty}^{\infty}\left(\frac{1}{2}-v^{2}+u^{2}\right) \operatorname{erf}(u-|v|) e^{-u^{2}} \mathrm{~d} u .
$$

Although it is not at all straightforward because of the presence of the error function, we were able to proceed again by reducing the double integral (26) to a single one [see result (B10) in Appendix B]:

$$
\begin{aligned}
I_{2}= & \frac{4 \ell}{\pi^{3 / 2} \hbar}\left\{-2 \sqrt{\pi} \int_{0}^{\infty} e^{-v^{2}} \cos (2 q v) \operatorname{erf}\left(\frac{v}{\sqrt{2}}\right) \mathrm{d} v\right. \\
& \left.+2 \sqrt{\pi} \int_{0}^{\infty} v^{2} e^{-v^{2}} \cos (2 q v) \operatorname{erf}\left(\frac{v}{\sqrt{2}}\right) \mathrm{d} v+\frac{\sqrt{2}}{2} \int_{0}^{\infty} e^{-3 v^{2} / 2} v \cos (2 q v) \mathrm{d} v\right\} .
\end{aligned}
$$

Collecting the results (25) and (27), the momentum density (20) may be written as

$$
n_{D}(p)=\frac{8 \ell}{\pi^{3 / 2} \hbar}\left\{\sqrt{2} J_{A}-\sqrt{\pi} J_{\mathrm{B}}+\sqrt{\pi} J_{C}\right\},
$$

in terms of three single integrals

$$
\begin{aligned}
& J_{A}=\int_{0}^{\infty} e^{-3 v^{2} / 2} v \cos (2 q v) \mathrm{d} v \\
& J_{\mathrm{B}}=\int_{0}^{\infty} e^{-v^{2}} \cos (2 q v) \operatorname{erf}\left(\frac{v}{\sqrt{2}}\right) \mathrm{d} v,
\end{aligned}
$$




$$
J_{C}=\int_{0}^{\infty} e^{-v^{2}} v^{2} \cos (2 q v) \operatorname{erf}\left(\frac{v}{\sqrt{2}}\right) \mathrm{d} v .
$$

We proceed now with their evaluation.

For $J_{A}$, we first perform an integration by parts to write

$$
J_{A}=\frac{1}{3}\left[1-2 q \int_{0}^{\infty} e^{-3 v^{2} / 2} \sin (2 q v) \mathrm{d} v\right] .
$$

We then apply the identity (see eq. (3.896), number (3) of ref. [19])

$$
\int_{0}^{\infty} e^{-\alpha x^{2}} \sin (\beta x) \mathrm{d} x=\frac{\beta}{2 \alpha} M\left(1, \frac{3}{2} ;-\frac{\beta^{2}}{4 \alpha}\right),
$$

where $M(a, b ; z) \equiv{ }_{1} F_{1}(a, b ; z)$ is the confluent hypergeometric function defined by [19]

$$
M(a, b ; z)=\sum_{n=0}^{\infty} \frac{(a)_{n}}{(b)_{n}} \frac{z^{n}}{n !},
$$

being $(a)_{n}=a(a+1)(a+2) \ldots(a+n-1)=\Gamma(a+n) / \Gamma(a)$ the Pochhammer symbol. We thus get

$$
J_{A}=\frac{1}{3}\left[1-\frac{4}{3} q^{2} M\left(1, \frac{3}{2} ;-\frac{2}{3} q^{2}\right)\right] .
$$

For $J_{\mathrm{B}}$, we first replace $\cos (x)=\left(e^{i x}+e^{-i x}\right) / 2$, and then use the tabulated relation [21]

$$
\begin{aligned}
\int_{0}^{\infty} x^{\alpha-1} e^{-\beta x} e^{-\gamma x^{2}}(\varepsilon x) \mathrm{d} x= & \frac{\varepsilon}{\sqrt{\pi} \gamma^{\frac{\alpha+1}{2}}} \Gamma\left(\frac{\alpha+1}{2}\right) \Psi_{1}\left(\frac{\alpha+1}{2}, \frac{1}{2}, \frac{3}{2}, \frac{1}{2} ;-\frac{\varepsilon^{2}}{\gamma}, \frac{\beta^{2}}{4 \gamma}\right) \\
& -\frac{\varepsilon \beta}{\sqrt{\pi} \gamma^{\frac{\alpha}{2}+1}} \Gamma\left(\frac{\alpha}{2}+1\right) \Psi_{1}\left(\frac{\alpha}{2}+1, \frac{1}{2}, \frac{3}{2}, \frac{3}{2} ;-\frac{\varepsilon^{2}}{\gamma}, \frac{\beta^{2}}{4 \gamma}\right),
\end{aligned}
$$

where $\Psi_{1}\left(a, b, c_{1}, c_{2} ; z_{1}, z_{2}\right)$ is the two-variable (degenerate) confluent hypergeometric series [21,22], given by [23]:

$$
\begin{aligned}
\Psi_{1}\left(a, b, c_{1}, c_{2} ; z_{1}, z_{2}\right) & =\sum_{m=0}^{\infty} \sum_{n=0}^{\infty} \frac{(a)_{m+n}(b)_{m}}{\left(c_{1}\right)_{m}\left(c_{2}\right)_{n}} \frac{z_{1}^{m}}{m !} \frac{z_{2}^{n}}{n !}=\sum_{m=0}^{\infty} \frac{(a)_{m}(b)_{m}}{\left(c_{1}\right)_{m}} \frac{z_{1}^{m}}{m !} M\left(a+m, c_{2} ; z_{2}\right) \\
& =\sum_{n=0}^{\infty} \frac{(a)_{n}}{\left(c_{2}\right)_{n}} \frac{z_{2}^{n}}{n !}{ }_{2} F_{1}\left(a+n, b, c_{1 ;} z_{1}\right), \quad\left|z_{1}\right|<1
\end{aligned}
$$

where ${ }_{2} F_{1}(a, b, c ; z)=\sum_{n=0}^{\infty}\left((a)_{n}(b)_{n} /(c)_{n}\right) z^{n} / n !$ is the Gaussian hypergeometric function. In our case $\alpha=1, \beta=\mp i q, \gamma=1 / 4, \varepsilon=1 /(2 \sqrt{2})$, and replacing $q=\ell p / \hbar$, we have

$$
\begin{aligned}
J_{\mathrm{B}} & =\frac{1}{2} \int_{0}^{\infty} e^{-\frac{v^{2}}{4}} e^{i q v} \operatorname{erf}\left(\frac{v}{2 \sqrt{2}}\right) \mathrm{d} v+\frac{1}{2} \int_{0}^{\infty} e^{-\frac{v^{2}}{4}} e^{-i q v} \operatorname{erf}\left(\frac{v}{2 \sqrt{2}}\right) \mathrm{d} v \\
& =\frac{1}{2} \sqrt{\frac{2}{\pi}} \Psi_{1}\left(1, \frac{1}{2}, \frac{3}{2}, \frac{1}{2} ;-\frac{1}{2}, \frac{-\ell^{2} p^{2}}{\hbar^{2}}\right) .
\end{aligned}
$$


The evaluation of $J_{C}$ is similar with $\alpha=3$ instead of $\alpha=1$

$$
J_{C}=\frac{1}{2} \sqrt{\frac{2}{\pi}} \Psi_{1}\left(2, \frac{1}{2}, \frac{3}{2}, \frac{1}{2} ;-\frac{1}{2},-\frac{\ell^{2} p^{2}}{\hbar^{2}}\right) .
$$

Note that $J_{\mathrm{B}}$ and $J_{C}$ converge as functions of the momentum $p$, as in both cases the function $\Psi_{1}$ has $z_{1}=-1 / 2$, which is within the convergence interval $\left|z_{1}\right|<1$.

Hence, collecting the results, we find

$$
\begin{aligned}
& n_{D}(p)=\frac{4 \ell}{\pi \hbar} \sqrt{\frac{2}{\pi}}\left\{\Psi_{1}\left(2, \frac{1}{2}, \frac{3}{2}, \frac{1}{2} ;-\frac{1}{2},-\frac{\ell^{2} p^{2}}{\hbar^{2}}\right)-\Psi_{1}\left(1, \frac{1}{2}, \frac{3}{2}, \frac{1}{2} ;-\frac{1}{2},-\frac{\ell^{2} p^{2}}{\hbar^{2}}\right)\right. \\
& \left.\frac{2}{3}\left[1-\frac{4}{3}\left(\frac{\ell p}{\hbar}\right)^{2} M\left(1, \frac{3}{2} ;-\frac{2}{3} \frac{\ell^{2} p^{2}}{\hbar^{2}}\right)\right]\right\} \text {. }
\end{aligned}
$$

\subsection{Closed-form expression of the momentum distribution $n_{\mathrm{B}}(p)$}

Collecting $n_{F}(p)$ from (23) and the result in (41), we obtain the closed-form expression of the momentum distribution

$$
\begin{aligned}
n_{\mathrm{B}}(p)= & \frac{\ell}{\sqrt{\pi} \hbar}\left[1+\frac{2 \ell^{2} p^{2}}{\hbar^{2}}\right] e^{\frac{-\ell^{2} p^{2}}{\hbar^{2}}} \\
& +\frac{\ell}{\sqrt{\pi} \hbar}\left(\frac{4 \sqrt{2}}{\pi}\right)\left\{\frac{2}{3}\left[1-\frac{4}{3}\left(\frac{\ell p}{\hbar}\right)^{2} M\left(1, \frac{3}{2} ;-\frac{2}{3} \frac{\ell^{2} p^{2}}{\hbar^{2}}\right)\right]\right. \\
& \left.+\Psi_{1}\left(2, \frac{1}{2}, \frac{3}{2}, \frac{1}{2} ;-\frac{1}{2},-\frac{\ell^{2} p^{2}}{\hbar^{2}}\right)-\Psi_{1}\left(1, \frac{1}{2}, \frac{3}{2}, \frac{1}{2} ;-\frac{1}{2},-\frac{\ell^{2} p^{2}}{\hbar^{2}}\right)\right\},
\end{aligned}
$$

which is the principal result of our paper. In the next section, we provide strong tests that support its validity.

We have computed $n_{\mathrm{B}}(p)$ for any value of $p$ using either of the two series representations (37) or (38). For the evaluation of the $\Psi_{1}$ function, from a numerical convergence point of view it turns out that the series in terms of confluent hypergeometric functions is more convenient because $\left|z_{1}\right|<1$ while $\left|z_{2}\right|$ can take any positive value. The numerical results for $n_{\mathrm{B}}(p)$ have been further confirmed by using (28) and direct numerical integration of (29), (30) and (31). A plot of $\hbar n_{\mathrm{B}} / \ell$ as a function of $q=\ell p / \hbar$ is shown in Fig. 1, and is compared to both contributions $n_{F}$ and $n_{D}$. We clearly observe a substantial shape difference between the fermionic and bosonic cases. The fermionic distribution presents a minimum at $q=0$, a maximum at $q=1 / \sqrt{2}$, and decreases rapidly to zero due to the $e^{-q^{2}}$ term. The bosonic distribution, on the other hand, has almost double value at $q=0$ (see ratio $n_{\mathrm{B}}(0) / n_{F}(0) \approx 2.02$ discussed in Sect. 4.1$)$, and decreases thereafter but in much slower fashion; approximately beyond $q>3$, we have $n_{\mathrm{F}} \simeq 0$ and $n_{\mathrm{B}}$ is essentially due to the deviation $n_{D}$.

\section{Tests of the momentum distribution expression (42)}

We consider now three tests on the analytical expression of the momentum distribution $n_{\mathrm{B}}(p)$. First, we will verify that the correct value of $n_{\mathrm{B}}(p=0)$ is obtained, then we will check the normalization condition, and finally we will make sure that we recover the correct high- $p$ asymptotic behavior. 


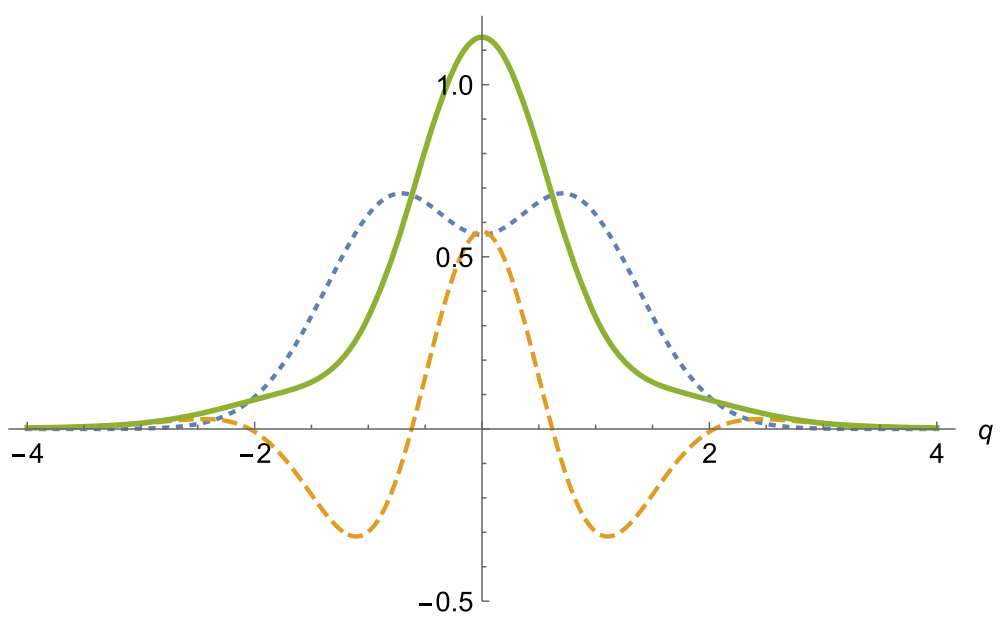

Fig. 1 Plot of $n_{\mathrm{B}}(p) \hbar / \ell$ as a function of $q=\ell p / \hbar$ (green solid curve) as given by (42). We also display $n_{F}(p) \hbar / \ell$ in (23) (blue dotted curve) and their difference $n_{D}(p) \hbar / \ell(41)$ (orange dashed curve)

\subsection{Test of $n_{\mathrm{B}}(p)$ at zero momentum $p=0$}

We wish to compare the value of momentum density $n_{\mathrm{B}}(p)$ at $p=0$ by using its definition in (16) with that obtained through our general expression (42). While the idea of the test is simple, the calculations are not straightforward.

Using the definition (16) together with $\rho_{\mathrm{B}}\left(x_{1}, x_{2}\right)$ given by the first equality in (7), gives for $p=0$

$$
\begin{aligned}
n_{\mathrm{B}}(0) & =\frac{1}{2 \pi \hbar} \int_{-\infty}^{\infty} \int_{-\infty}^{\infty} \rho_{\mathrm{B}}\left(x_{1}, x_{2}\right) \mathrm{d} x_{1} \mathrm{~d} x_{2} \\
& =\frac{\ell}{\pi^{2} \hbar} \int_{-\infty}^{\infty} \int_{-\infty}^{\infty} e^{-\frac{\left(z_{1}^{2}+z_{2}^{2}\right)}{2}} d z_{1} d z_{2} \int_{-\infty}^{\infty} e^{-\xi^{2}}\left|z_{1}-\xi\right|\left|z_{2}-\xi\right| \mathrm{d} \xi .
\end{aligned}
$$

For any fixed value of $\xi$, we make the change of variables $z_{1}=r+\xi, z_{2}=s+\xi$, and get

$$
n_{\mathrm{B}}(0)=\frac{\ell}{\pi^{2} \hbar} \int_{-\infty}^{\infty} \int_{-\infty}^{\infty}|r||s| e^{-\frac{\left(r^{2}+s^{2}\right)}{2}} \mathrm{~d} r \mathrm{~d} s \int_{-\infty}^{\infty} e^{-2 \xi^{2}} e^{-(r+s) \xi} \mathrm{d} \xi
$$

After the integration on $\xi$ is performed, the above expression reduces to

$$
\begin{aligned}
n_{\mathrm{B}}(0) & =\frac{\ell}{\pi^{3 / 2} \sqrt{2} \hbar} \int_{-\infty}^{\infty} \mathrm{d} r|r| e^{-\frac{3}{8} r^{2}} \int_{-\infty}^{\infty} d s|s| e^{-\frac{3}{8} s^{2}} e^{\frac{r s}{4}} \\
& =\frac{\ell \sqrt{2}}{\pi^{3 / 2} \hbar} \int_{-\infty}^{\infty} \mathrm{d} r|r| e^{-\frac{3}{8} r^{2}} \int_{0}^{\infty} s e^{-\frac{3}{8} s^{2}} \cosh \left(\frac{r s}{4}\right) \mathrm{d} s .
\end{aligned}
$$

Using the identity (see eq. (3.562) number (4) of [19])

$$
\int_{0}^{\infty} s e^{-\beta s^{2}} \cosh (\gamma s) \mathrm{d} s=\frac{1}{2 \beta}+\frac{\gamma}{4 \beta} \sqrt{\frac{\pi}{\beta}} e^{\frac{\gamma^{2}}{4 \beta}} \operatorname{erf}\left(\frac{\gamma}{2 \sqrt{\beta}}\right)
$$

for $\beta=3 / 8, \gamma=r / 4$ in (45), we obtain

$$
n_{\mathrm{B}}(0)=\frac{\ell \sqrt{2}}{\pi^{3 / 2} \hbar} \int_{-\infty}^{\infty}|r| e^{-\frac{3}{8} r^{2}} \mathrm{~d} r\left[\frac{4}{3}+\sqrt{\frac{8 \pi}{3}} e^{\frac{r^{2}}{24}} \frac{r}{6} \operatorname{erf}\left(\frac{r}{4} \sqrt{\frac{2}{3}}\right)\right]
$$




$$
=\frac{\ell \sqrt{2}}{\pi^{3 / 2} \hbar}\left[\frac{32}{9}+\sqrt{\frac{8 \pi}{27}} \int_{0}^{\infty} r^{2} e^{-\frac{r^{2}}{3}} \operatorname{erf}\left(\frac{r}{4} \sqrt{\frac{2}{3}}\right)\right] .
$$

The remaining integral can be found in the literature [24], and after simplifications we get

$$
n_{\mathrm{B}}(0)=\frac{\ell}{\sqrt{\pi} \hbar}\left[\frac{4}{\pi} \arctan (\sqrt{2})+\frac{4 \sqrt{2}}{\pi}-1\right] .
$$

It is interesting to compare this value of the momentum density at $p=0$ (peak momentum distribution) with its two-fermions system counterpart given by (23)

$$
n_{F}(0)=\frac{\ell}{\sqrt{\pi} \hbar} .
$$

We clearly observe a large deviation measured by the ratio $n_{\mathrm{B}}(0) / n_{F}(0)$ $=\left[\frac{4}{\pi} \arctan (\sqrt{2})+\frac{4 \sqrt{2}}{\pi}-1\right] \approx 2.02$. This ratio is featured in Fig. 1 .

Let us now check that the result (47) is also obtained by setting $p=0$ into expression (42)

$$
n_{\mathrm{B}}(0)=\frac{\ell}{\sqrt{\pi} \hbar}+\frac{\ell}{\sqrt{\pi} \hbar}\left(\frac{4 \sqrt{2}}{\pi}\right)\left[\frac{2}{3}+\Psi_{1}\left(2, \frac{1}{2}, \frac{3}{2}, \frac{1}{2} ;-\frac{1}{2}, 0\right)-\Psi_{1}\left(1, \frac{1}{2}, \frac{3}{2}, \frac{1}{2} ;-\frac{1}{2}, 0\right)\right] .
$$

In order to compute $\Psi_{1}\left(1, \frac{1}{2}, \frac{3}{2}, \frac{1}{2} ;-\frac{1}{2}, 0\right)$ and $\Psi_{1}\left(2, \frac{1}{2}, \frac{3}{2}, \frac{1}{2} ;-\frac{1}{2}, 0\right)$, we return to the third form of the confluent hypergeometric function with two variables in (38) and set $z_{2}=0$ :

$$
\begin{aligned}
& \Psi_{1}\left(1, \frac{1}{2}, \frac{3}{2}, \frac{1}{2} ;-\frac{1}{2}, 0\right)={ }_{2} F_{1}\left(1, \frac{1}{2}, \frac{3}{2} ;-\frac{1}{2}\right), \\
& \Psi_{1}\left(2, \frac{1}{2}, \frac{3}{2}, \frac{1}{2} ;-\frac{1}{2}, 0\right)={ }_{2} F_{1}\left(2, \frac{1}{2}, \frac{3}{2} ;-\frac{1}{2}\right) .
\end{aligned}
$$

To compute ${ }_{2} F_{1}\left(1, \frac{1}{2}, \frac{3}{2} ;-\frac{1}{2}\right)$ we use the relation ${ }_{2} F_{1}\left(1, \frac{1}{2}, \frac{3}{2} ;-z^{2}\right)=(\arctan z) / z($ see (9.121) number (27) of ref. [19]), with $z^{2}=1 / \sqrt{2}$. Making use of the well-known relation $\arctan (z)+\arctan (1 / z)=\pi / 2$, we find

$$
{ }_{2} F_{1}\left(1, \frac{1}{2}, \frac{3}{2} ;-\frac{1}{2}\right)=\sqrt{2} \arctan (1 / \sqrt{2})=\sqrt{2}\left[\frac{\pi}{2}-\arctan (\sqrt{2})\right] .
$$

The other quantity ${ }_{2} F_{1}\left(2, \frac{1}{2}, \frac{3}{2} ;-\frac{1}{2}\right)$ can be easily obtained by applying the recursion relation (15.2.14) of [25] with $a=1, b=1 / 2$ and $c=3 / 2$ :

$$
\begin{aligned}
{ }_{2} F_{1}\left(2, \frac{1}{2}, \frac{3}{2} ;-\frac{1}{2}\right) & =\frac{1}{2}{ }_{2} F_{1}\left(1, \frac{1}{2}, \frac{3}{2} ;-\frac{1}{2}\right)+\frac{1}{2}{ }_{2} F_{1}\left(1, \frac{3}{2}, \frac{3}{2} ;-\frac{1}{2}\right) \\
& =\frac{1}{2}{ }_{2} F_{1}\left(1, \frac{1}{2}, \frac{3}{2} ;-\frac{1}{2}\right)+\frac{1}{3}
\end{aligned}
$$

where the second equality is obtained by noting that ${ }_{2} F_{1}(1, b, b ; z)=1 /(1-z)$, here with $z=-1 / 2$. Substituting (50), together with (51) and (52), into (49), we recover the peak momentum distribution value (47), thus ending the proof. 
4.2 Normalization condition of momentum distribution $n_{\mathrm{B}}(p)$

Next, we want to show that momentum density obeys the normalization condition $\int n_{\mathrm{B}}(p) \mathrm{d} p=2$. It is more convenient not to use the form of $n_{\mathrm{B}}(p)$ in (42), but an expression obtained a few steps before. That is, we use the momentum density as given in (18), $n_{\mathrm{B}}(p)=n_{F}(p)+n_{D}(p)$, with the expressions for $n_{F}(p)$ and $n_{D}(p)$ given by (21) and (28), to write

$$
\begin{gathered}
n_{\mathrm{B}}(p)=\frac{2 \ell}{\hbar \pi}\left[\int_{-\infty}^{\infty} e^{-v^{2}} \cos (2 q v) \mathrm{d} v-\int_{-\infty}^{\infty} v^{2} e^{-v^{2}} \cos (2 q v) \mathrm{d} v\right] \\
+\frac{4 \ell}{\pi^{3 / 2} \hbar}\left[\sqrt{2} \int_{-\infty}^{\infty} e^{-\frac{3 v^{2}}{2}}|v| \cos (2 q v) \mathrm{d} v\right. \\
-\sqrt{\pi} \int_{-\infty}^{\infty} e^{-v^{2}} \cos (2 q v) \operatorname{erf}\left(\frac{|v|}{\sqrt{2}}\right) \mathrm{d} v \\
\left.+\sqrt{\pi} \int_{-\infty}^{\infty} e^{-v^{2}} v^{2} \cos (2 q v) \operatorname{erf}\left(\frac{|v|}{\sqrt{2}}\right) \mathrm{d} v\right] .
\end{gathered}
$$

Taking into account the following representation of the Dirac delta distribution

$$
\int_{-\infty}^{\infty} \cos (2 q v) \mathrm{d} q=\pi \delta(v)
$$

the integral over the momentum $p$ (or equivalently over $q=\ell p / \hbar$ ) is quite straightforward

$$
\begin{aligned}
\int n_{\mathrm{B}}(p) \mathrm{d} p= & 2 \int_{-\infty}^{\infty}\left[e^{-v^{2}}-v^{2} e^{-v^{2}}+\frac{2 \sqrt{2}}{\sqrt{\pi}} e^{-\frac{3 v^{2}}{2}}|v|\right. \\
& \left.-2 e^{-v^{2}} \operatorname{erf}\left(\frac{|v|}{\sqrt{2}}\right)+2 e^{-v^{2}} v^{2} \operatorname{erf}\left(\frac{|v|}{\sqrt{2}}\right)\right] \delta(v) \mathrm{d} v=2,
\end{aligned}
$$

where the final result is obtained using $\operatorname{erf}(0)=0$. We have thus checked that, for the twoparticle system considered in this work, we have the correct normalization condition for $n_{\mathrm{B}}(p)$.

\subsection{High- $p$ behavior of the momentum distribution and Tan's coefficient}

Here, we will examine the asymptotic behavior of the momentum density $n_{\mathrm{B}}(p)$ obtained in (42) as $p \rightarrow \infty$ and we will prove that

$$
n_{\mathrm{B}}(p) \approx \frac{C_{2}}{p^{4}}, \quad p \rightarrow \infty, \quad C_{2}=(2 m \hbar \omega / \pi)^{3 / 2}=(2 / \pi)^{3 / 2}(\hbar / \ell)^{3},
$$

where $C_{2}$ is the exact Tan's contact coefficient for the two-atom system under study [14] (for a recent study of this coefficient see [26,27]) recovering the $1 / p^{4}$ dependence originated from the short-range character of the interaction.

To derive this result, we search the asymptotic behavior up to $1 / p^{4}$. For this purpose, we need the asymptotic behavior $(x \rightarrow+\infty)$ [28]

$$
M(a, b ;-x) \approx \frac{\Gamma(b)}{\Gamma(b-a)} x^{-a} \sum_{k=0}^{\infty} \frac{(1+a-b)_{k}(a)_{k}}{k !} x^{-k},
$$

$a \neq 0,-1,-2, \ldots$ 
Taking $a=1, b=\frac{3}{2}$ and $x=2 \ell^{2} p^{2} /\left(3 \hbar^{2}\right)$, we have from (57) that

$$
\begin{aligned}
\frac{4}{3}\left(\frac{\ell p}{\hbar}\right)^{2} M\left(1, \frac{3}{2} ;-\frac{2}{3} \frac{\ell^{2} p^{2}}{\hbar^{2}}\right) & \approx \sum_{k=0}^{\infty} \frac{\left(\frac{1}{2}\right)_{k}(1)_{k}}{k !}\left(\frac{3 \hbar^{2}}{2 \ell^{2} p^{2}}\right)^{k} \\
& =1+\frac{3}{4} \frac{\hbar^{2}}{\ell^{2} p^{2}}+\frac{27}{16} \frac{\hbar^{4}}{\ell^{4} p^{4}}+O\left(\frac{1}{p^{6}}\right) .
\end{aligned}
$$

To determine the asymptotic behavior of $\Psi_{1}\left(1+j, 1 / 2,3 / 2,1 / 2 ;-1 / 2,-\ell^{2} p^{2} / \hbar^{2}\right)$ as $p \rightarrow \infty$, with either $j=0$ or $j=1$, we use the second expression in (37) to write

$$
\begin{aligned}
\Psi_{1}\left(1+j, \frac{1}{2}, \frac{3}{2}, \frac{1}{2} ;-\frac{1}{2},-\frac{\ell^{2} p^{2}}{\hbar^{2}}\right)= & \sum_{m=0}^{\infty} \frac{(1+j)_{m}\left(\frac{1}{2}\right)_{m}}{\left(\frac{3}{2}\right)_{m}} \frac{\left(-\frac{1}{2}\right)^{m}}{m !} \\
& \times M\left(1+j+m, \frac{1}{2} ;-\frac{\ell^{2} p^{2}}{\hbar^{2}}\right)
\end{aligned}
$$

and then the asymptotic expansion (57) to get

$$
\begin{aligned}
\Psi_{1}\left(1+j, \frac{1}{2}, \frac{3}{2}, \frac{1}{2} ;-\frac{1}{2},-\frac{\ell^{2} p^{2}}{\hbar^{2}}\right) \approx & \sum_{m=0}^{\infty} \frac{(1+j)_{m}\left(\frac{1}{2}\right)_{m}}{\left(\frac{3}{2}\right)_{m}} \frac{\left(-\frac{1}{2}\right)^{m}}{m !} \frac{\Gamma\left(\frac{1}{2}\right)}{\Gamma\left(-m-j-\frac{1}{2}\right)} \\
& \times\left(\frac{\ell^{2} p^{2}}{\hbar^{2}}\right)^{-m-j-1} \sum_{k=0}^{\infty} \frac{\left(m+j+\frac{3}{2}\right)_{k}(m+j+1)_{k}}{k !}\left(\frac{\ell^{2} p^{2}}{\hbar^{2}}\right)^{-k} .
\end{aligned}
$$

To retain only terms up to $1 / p^{4}$, we have to consider the summation indexes up to $m+k \leq$ $1-j$. It is easy to show that

$$
\begin{aligned}
& \Psi_{1}\left(1, \frac{1}{2}, \frac{3}{2}, \frac{1}{2} ;-\frac{1}{2},-\frac{\ell^{2} p^{2}}{\hbar^{2}}\right) \approx-\frac{\hbar^{2}}{2 \ell^{2} p^{2}}-\frac{7}{8} \frac{\hbar^{4}}{\ell^{4} p^{4}}+O\left(\frac{1}{p^{6}}\right) \\
& \Psi_{1}\left(2, \frac{1}{2}, \frac{3}{2}, \frac{1}{2} ;-\frac{1}{2},-\frac{\ell^{2} p^{2}}{\hbar^{2}}\right) \approx \frac{3}{4} \frac{\hbar^{4}}{\ell^{4} p^{4}}+O\left(\frac{1}{p^{6}}\right) .
\end{aligned}
$$

Collecting expressions (58), (61) and (62) into (42), after the cancellation of the terms of order $1 / p^{2}$, one finds

$$
n_{\mathrm{B}}(p) \approx\left(\frac{2 \hbar^{3} \sqrt{2}}{\ell^{3} \pi^{\frac{3}{2}}}\right) \frac{1}{p^{4}}=\left(\frac{2 m \hbar \omega}{\pi}\right)^{\frac{3}{2}} \frac{1}{p^{4}},
$$

which is the result announced in (56): we have recovered the well-known $1 / p^{4}$ asymptotic decay of $n_{\mathrm{B}}(p)$ for high momentum values. The coefficient in front of the high- $p$ tail of the momentum distribution, called Tan's coefficient [14], is given by $(2 / \pi)^{3 / 2}(m \omega \hbar)^{3 / 2}$ for the system of two particles under study. This coefficient can be found in the literature $[5,29$, 30], possibly in slightly different forms according to the way the momentum distribution is normalized, and whether $p$ or $k=p / \hbar$ is used as variable for the momentum density.

The high- $p$ tail behavior can be observed in Fig. 2 where we show $n_{\mathrm{B}}(p), n_{F}(p)$ and $n_{D}(p)$ multiplied by $p^{4} / C_{2}$. One can appreciate how $n_{\mathrm{B}}(p)$ tends to 1 for large $q$, and that the asymptotic limit is dominated by the deviation term $n_{D}$, since the $n_{\mathrm{F}}$ is already negligible for $q>4$. 


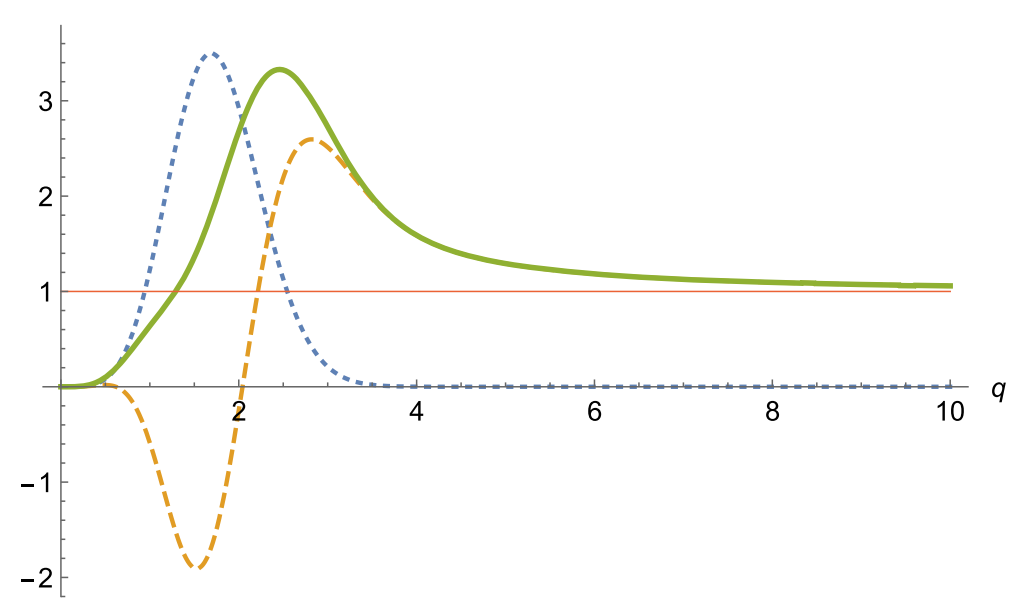

Fig. 2 Plot of $n_{\mathrm{B}}(p) p^{4} / C_{2}$ (green solid curve), $n_{F}(p) p^{4} / C_{2}$ (blue dotted curve) and $n_{D}(p) p^{4} / C_{2}$ (yellow dashed curve) as a function of $q=\ell p / \hbar$, with $C_{2}=(2 / \pi)^{3 / 2}(\hbar / \ell)^{3}$

\section{Conclusion}

In this paper, we have studied a one-dimensional quantum system consisting of two bosons harmonically trapped and with an infinite contact repulsion. This system has been the subject of numerous studies, in particular with respect to its asymptotic momentum distribution. In the present work, we have been able to provide an exact expression of the momentum distribution valid for arbitrary value of the momentum, and thus, we have extended the list of exactly solvable models of two interacting particles. Starting from the wave function of the two particles, we have derived an exact expression of the one-body density matrix expressed in terms of center of mass and relative coordinates. From this result, we have calculated the corresponding momentum distribution. To clearly identify the deviation of this system from the two noninteracting fermions, we wrote this momentum density as the sum of two terms: a first one corresponding to the two noninteracting fermions, and a second one related to the short-range character of the interaction.

In order to validate our analytic expression of the momentum density, three robust tests have been performed satisfactorily. In particular, the third test concerns the asymptotic decay for high momentum values; we have been able to recover the exact expression of the Tan contact coefficient, which we recall is the coefficient in front of the high- $p$ tail of the momentum distribution, for the case of two particles [14].

The analytical advances made in the present work starting from the ground state wave function open the door to deal with the excited states of the system in question. The time evolution of the excited states of two identical TG bosons was recently examined in nonequilibrium quantum dynamics, a configuration generated by a sudden switch-off in the interaction strength [12]. Within the present analysis, it may be of interest to extend the study and consider the resulting temporal evolution of the density matrix and the momentum distribution.

Acknowledgements This research was funded by Junta de Castilla y León (Spain) and FEDER (project BU229P18), and by the Directorate General for Scientific Research and Technological Development (DGRSDT) of Algeria. 
Funding Open Access funding provided thanks to the CRUE-CSIC agreement with Springer Nature.

Open Access This article is licensed under a Creative Commons Attribution 4.0 International License, which permits use, sharing, adaptation, distribution and reproduction in any medium or format, as long as you give appropriate credit to the original author(s) and the source, provide a link to the Creative Commons licence, and indicate if changes were made. The images or other third party material in this article are included in the article's Creative Commons licence, unless indicated otherwise in a credit line to the material. If material is not included in the article's Creative Commons licence and your intended use is not permitted by statutory regulation or exceeds the permitted use, you will need to obtain permission directly from the copyright holder. To view a copy of this licence, visit http://creativecommons.org/licenses/by/4.0/.

\section{Appendix A}

In this Appendix, we will calculate the two functions $\mathcal{B}(u, v)$ and $\mathcal{C}(u, v)$ given by (11), and thereafter $\mathcal{A}(u, v)$ defined in (10). Making the change of variable $t=w+u$ in $\mathcal{B}(u, v)$, we get

$$
\mathcal{B}(u, v)=\int_{-\infty}^{u-|v|} t^{2} e^{-t^{2}} \mathrm{~d} t-2 u \int_{-\infty}^{u-|v|} t e^{-t^{2}} \mathrm{~d} t+\left(u^{2}-v^{2}\right) \int_{-\infty}^{u-|v|} e^{-t^{2}} \mathrm{~d} t .
$$

If we perform an integration by parts in the first integral, we then get after rearrangements

$$
\mathcal{B}(u, v)=\frac{1}{2}(u+|v|) e^{-(u-|v|)^{2}}+\left(\frac{1}{2}+u^{2}-v^{2}\right) \int_{-\infty}^{u-|v|} e^{-t^{2}} \mathrm{~d} t .
$$

Proceeding similarly for $\mathcal{C}(u, v)$, we write

$$
\mathcal{C}(u, v)=\int_{u-|v|}^{u+|v|} t^{2} e^{-t^{2}} \mathrm{~d} t-2 u \int_{u-|v|}^{u+|v|} t e^{-t^{2}} \mathrm{~d} t+\left(u^{2}-v^{2}\right) \int_{u-|v|}^{u+|v|} e^{-t^{2}} \mathrm{~d} t .
$$

Carrying out an integration by parts of the first integral, transforms (A3) as

$\mathcal{C}(u, v)=\frac{1}{2}(u-|v|) e^{-(u+|v|)^{2}}-\frac{1}{2}(u+|v|) e^{-(u-|v|)^{2}}+\left(\frac{1}{2}+u^{2}-v^{2}\right) \int_{u-|v|}^{u+|v|} e^{-t^{2}} \mathrm{~d} t$.

Collecting the two results (A2) and (A4), the expression of $\mathcal{A}(u, v)$ in (10) becomes

$$
\begin{aligned}
\mathcal{A}(u, v)= & (u+|v|) e^{-(u-|v|)^{2}}-(u-|v|) e^{-(u+|v|)^{2}} \\
& +\left(\frac{1}{2}+u^{2}-v^{2}\right)\left[\int_{-\infty}^{u-|v|} e^{-t^{2}} \mathrm{~d} t+\int_{-\infty}^{-u-|v|} e^{-t^{2}} \mathrm{~d} t-\int_{u-|v|}^{u+|v|} e^{-t^{2}} \mathrm{~d} t\right] .
\end{aligned}
$$

To further simplify, let us observe that

$$
\int_{-\infty}^{u-|v|} e^{-t^{2}} \mathrm{~d} t+\int_{-\infty}^{-u-|v|} e^{-t^{2}} \mathrm{~d} t=\int_{-\infty}^{\infty} e^{-t^{2}} \mathrm{~d} t-\int_{u-|v|}^{u+|v|} e^{-t^{2}} \mathrm{~d} t=\sqrt{\pi}-\int_{u-|v|}^{u+|v|} e^{-t^{2}} \mathrm{~d} t .
$$

Using the definition of the erf function $\int_{0}^{a} e^{-t^{2}} \mathrm{~d} t=\frac{1}{2} \sqrt{\pi} \operatorname{erf}(a)$, we further have

$$
\int_{u-|v|}^{u+|v|} e^{-t^{2}} \mathrm{~d} t=\frac{\sqrt{\pi}}{2}(\operatorname{erf}(u+|v|)-\operatorname{erf}(u-|v|)) .
$$

Combining the above two results, the expression of $\mathcal{A}(u, v)$ becomes 


$$
\begin{aligned}
\mathcal{A}(u, v)= & (u+|v|) e^{-(u-|v|)^{2}}-(u-|v|) e^{-(u+|v|)^{2}} \\
& +\sqrt{\pi}\left(\frac{1}{2}+u^{2}-v^{2}\right)(1+\operatorname{erf}(u-|v|)-\operatorname{erf}(u+|v|)) .
\end{aligned}
$$

\section{Appendix B}

In this Appendix, we consider the double integral $I_{2}$ in (26)

$$
\begin{aligned}
I_{2} & =\frac{4 \ell}{\pi^{3 / 2} \hbar} \int_{-\infty}^{\infty} e^{-v^{2}} \cos (2 q v) \mathrm{d} v \int_{-\infty}^{\infty}\left(\frac{1}{2}-v^{2}+u^{2}\right) \operatorname{erf}(u-|v|) e^{-u^{2}} \mathrm{~d} u \\
& =\frac{4 \ell}{\pi^{3 / 2} \hbar}\left(K_{1}+K_{2}+K_{3}\right),
\end{aligned}
$$

which, for convenience, we have split into three double integrals defined through

$$
\begin{aligned}
& K_{1}=\frac{1}{2} \int_{-\infty}^{\infty} e^{-v^{2}} \cos (2 q v) \mathrm{d} v \int_{-\infty}^{\infty} e^{-u^{2}} \operatorname{erf}(u-|v|) \mathrm{d} u, \\
& K_{2}=-\int_{-\infty}^{\infty} v^{2} e^{-v^{2}} \cos (2 q v) \mathrm{d} v \int_{-\infty}^{\infty} e^{-u^{2}} \operatorname{erf}(u-|v|) \mathrm{d} u, \\
& K_{3}=\int_{-\infty}^{\infty} e^{-v^{2}} \cos (2 q v) \mathrm{d} v \int_{-\infty}^{\infty} u^{2} e^{-u^{2}} \operatorname{erf}(u-|v|) \mathrm{d} u .
\end{aligned}
$$

In order to compute the $u$ integral in (B4), we first perform an integration by parts to get

$$
\begin{aligned}
G=\int_{-\infty}^{\infty} u^{2} e^{-u^{2}} \operatorname{erf}(u-|v|) \mathrm{d} u= & \frac{1}{2} \int_{-\infty}^{\infty} e^{-u^{2}} \operatorname{erf}(u-|v|) \mathrm{d} u \\
& +\frac{1}{\sqrt{\pi}} \int_{-\infty}^{\infty} e^{-u^{2}} u e^{-(u-|v|)^{2}} \mathrm{~d} u .
\end{aligned}
$$

The second integral on the right-hand side of (B5) can be carried out, giving

$$
G=\frac{1}{2} \int_{-\infty}^{\infty} e^{-u^{2}} \operatorname{erf}(u-|v|) \mathrm{d} u+\frac{|v|}{2 \sqrt{2}} e^{-|v|^{2} / 2} .
$$

Hence, substituting (B6) into (B4), one finds out that $K_{3}$ is related to $K_{1}$ through

$$
K_{3}=K_{1}+\frac{1}{\sqrt{2}} \int_{0}^{\infty} e^{-3 v^{2} / 2} v \cos (2 q v) \mathrm{d} v .
$$

To carry out the $u$ integrals in (B2)-(B3), we start from the identity

$$
\int_{-\infty}^{\infty} e^{-(x+a)^{2}} \operatorname{erf}(x) \mathrm{d} x=-\sqrt{\pi} \operatorname{erf}\left(\frac{a}{\sqrt{2}}\right)=\int_{-\infty}^{\infty} e^{-t^{2}} \operatorname{erf}(t-a) \mathrm{d} t .
$$

Using the above relation for $a=|v|$ allows us to write the expressions of (B2) and (B3) as single integrals, so that

$$
\begin{aligned}
& K_{1}=-\sqrt{\pi} \int_{0}^{\infty} e^{-v^{2}} \cos (2 q v) \operatorname{erf}\left(\frac{v}{\sqrt{2}}\right) \mathrm{d} v \\
& K_{2}=2 \sqrt{\pi} \int_{0}^{\infty} v^{2} e^{-v^{2}} \cos (2 q v) \operatorname{erf}\left(\frac{v}{\sqrt{2}}\right) \mathrm{d} v
\end{aligned}
$$


Finally adding the three results (B9) and (B7), we can write (B1) as

$$
\begin{aligned}
I_{2}= & \frac{4 \ell}{\pi^{3 / 2} \hbar}\left\{-2 \sqrt{\pi} \int_{0}^{\infty} e^{-v^{2}} \cos (2 q v) \operatorname{erf}\left(\frac{v}{\sqrt{2}}\right) \mathrm{d} v\right. \\
& \left.+2 \sqrt{\pi} \int_{0}^{\infty} v^{2} e^{-v^{2}} \cos (2 q v) \operatorname{erf}\left(\frac{v}{\sqrt{2}}\right) \mathrm{d} v+\frac{\sqrt{2}}{2} \int_{0}^{\infty} e^{-3 v^{2} / 2} v \cos (2 q v) \mathrm{d} v\right\} .
\end{aligned}
$$

\section{References}

1. T. Kinoshita, T. Wenger, D.S. Weiss, Science 305, 1125 (2004)

2. M.D. Girardeau, J. Math. Phys. 1, 516 (1960)

3. M.D. Girardeau, E.M. Wright, Phys. Rev. Lett. 84, 5691 (2000)

4. M.D. Girardeau, E.M. Wright, J.M. Triscari, Phys. Rev. A 63, (2001)

5. G.J. Lapeyre, M.D. Girardeau, E.M. Wright, Phys. Rev. A 66, (2002)

6. P.J. Forrester, N.E. Frankel, T.M. Garoni, N.S. Witte, Phys. Rev. A 67, (2003)

7. P. Capuzzi, P. Vignolo, Phys. Rev. A 101, 013633 (2020)

8. F. Deuretzbacher, D. Becker, J. Bjerlin, S.M. Reimann, L. Santos, Phys. Rev. A 90, 013611 (2014)

9. A.G. Volosniev, D.V. Fedorov, A.S. Jensen, M. Valiente, N.T. Zinner, Nat. Commun. 5, 5300 (2014)

10. A.G. Volosniev, D. Petrosyan, M. Valiente, D.V. Fedorov, A.S. Jensen, N.T. Zinner, Phys. Rev. A 91, $023620(2015)$

11. S.S. Alam, T. Skaras, Li. Yang, H. Pu, Dynamical Fermionization in One Dimensional Spinor Gases (2020). arXiv:2008.08383v1 [cond-mat.quant-gas]

12. L.M.A. Kehrberger, V.J. Bolsinger, P. Schmelcher, Phys. Rev. A 97, 013606 (2018)

13. M. Olshanii, V. Dunjko, Phys. Rev. Lett. 91, 090401 (2003)

14. S. Tan, Ann. Phys. 323, 2952 (2008); 323, 2971 (2008); 323, 2987 (2008)

15. R.M. Dreizler, E.K.U. Gross, Density Functional Theory: An Approach to the Quantum Many-Body Problem (Springer, Berlin, 1990)

16. R. Pezer, H. Buljan, Phys. Rev. Lett. 98, 240403 (2007)

17. R. Boumaza, Dynamical Properties of Quantum Gases Following a Sudden Change of Confining Potential, Doctoral Thesis (UFA, Sétif, 2019)

18. P. Vignolo, A. Minguzzi, Phys. Rev. Lett. 110, 020403 (2013)

19. I.S. Gradshteyn, I.M. Ryzhik, Table of Integrals, Series and Products (Academic Press, New York, 1994)

20. C. Cohen-Tannoudji, B. Diu, F. Laloë, Quantum Mechanics, Volume 1: Basic Concepts, Tools, and Applications (Wiley-VCH, Weinheim, 2019)

21. A.P. Prudnikov, U.I.A. Brychkov, O.I. Marichev, Integrals and series. Vol 2, Special functions (Gordon and Breach Science Publishers, New York, 1986)

22. H.M. Srivastava, P.W. Karlsson, Multiple Gaussian Hypergeometric Series (Ellis Horwood, Chichester, 1985)

23. L.U. Ancarani, J.A. Del Punta, G. Gasaneo, J. Math. Phys. 58, 073504 (2017)

24. E.W. Ng, M. Gell, A table of integrals of error functions, Journal of research of the National Bureau of Standards-B. Math. Sci. 73B, 1 (1969)

25. M. Abramowitz, I.A. Stegun, Handbook of Mathematical Functions (Dover, New York, 1972)

26. G. Lang, P. Vignolo, A. Minguzzi, Eur. Phys. J. Spec. Top. 226, 1583 (2017)

27. O.I. Pâţu, A. Klümper, Phys. Rev. A 96, 063612 (2017)

28. F.W.J. Olver, D.W. Lozier, R.F. Boisvert, C.W. Clark (eds.), NIST Handbook of Mathematical Functions (Cambridge University Press, Cambridge, 2010)

29. A. Minguzzi, P. Vignolo, M.P. Tosi, Phys. Lett. A 294, 222 (2002)

30. F.T. Sant'Ana, F. Hébert, V.G. Rousseau, M. Albert, P. Vignolo, Phys. Rev. A 100, 063608 (2019) 